\title{
Optimal Harvesting Under Resource Stock and Price Uncertainty
}

\author{
Luis Alvarez \\ Turku School of Economics and Business Administration and RUESG \\ and \\ Erkki Koskela \\ University of Helsinki, RUESG and HECER
}

Discussion Paper No. 31

November 2004

ISSN 1795-0562

HECER - Helsinki Center of Economic Research, P.O. Box 17 (Arkadiankatu 7), Fl-00014 University of Helsinki, FINLAND, Tel +358-9-191-28780, Fax +358-9-191-28781,

E-mail info-hecer@helsinki.fi, Internet www.hecer.fi 


\title{
Optimal Harvesting Under Resource Stock and Price Uncertainty*
}

\begin{abstract}
We analyze optimal harvesting policy and its determinants in a general framework with both stochastic price and stock dynamics. We state a set of weak conditions under which the optimal harvesting policy can be characterized by a single harvesting threshold and show that the value of the optimal harvesting and depletion policies can be expressed in the separable form according to which only the current price and the expected per capita growth rate affect the threshold, while under risk neutrality the volatility of price dynamics will have no effect. We also show that uncertainty makes waiting valuable and that optimal harvesting threshold is higher in the case where harvesting can be exercised only once than in the sequential case.
\end{abstract}

JEL Classification: G31,H25,D80.

Keywords: optimal harvesting, stochastic price and stock dynamics, single and sequential harvesting opportunity.

Luis H.R. Alvarez

Department of Economics,

Quantitative Methods in Management

Turku School of Economics and

Business Administration

FI-20500 Turku

FINLAND

e-mail:luis.alvarez@tukkk.fi
Erkki Koskela

Department of Economics

P.O.Box 17 (Arkadiankatu 7)

University of Helsinki

FI-00014 University of Helsinki

FINLAND

e-mail: erkki.koskela@helsinki.fi

* The research of Luis H.R. Alvarez has been supported by the Foundation for the Promotion of the Actuarial Profession and the Finnish Insurance Society. Erkki Koskela thanks the Yrjö Jahnsson Foundation. Both authors acknowledge the financial support from the Research Unit of Economic Structures and Growth (RUESG) at the University of Helsinki. 


\section{Introduction}

The optimal timing problems of a discrete action constitute an important class of stochastic control problems in economics and finance including optimal investment threshold decisions and optimal exercise rules for financial options (for textbook treatments of these issues, see Dixit and Pindyck (1994) and Björk (1998)). Optimal timing problems of this sort also arise and have been studied in environmental and renewable resource economics including forest economics. In this paper our focus is in renewable resource issues. First, we provide a brief survey of what has been done in this literature and after that we present a new research question concerning the rational management of a stochastically fluctuating renewable resource in the presence of stochastic price dynamics.

Pindyck (1984) has studied the implications of stochastic fluctuations in the renewable resource stock on the return required to keep a unit of stock in situ and thereby the relationship between the extraction and volatility of the renewable resource stock when harvesters are risk neutral. He shows that stochastic fluctuations affect the optimal extraction rate via different channels which run counter to each other. In Pindyck (2000) the real option theory is applied to explore the optimal environmental policy in a framework with two stochastic variables, one which captures uncertainty over future costs and benefits of reduced environmental degradation and the other that captures uncertainty about the evolution of an ecosystem, which interact with two irreversibilities, namely sunk costs associated with environmental regulation and sunk benefits of avoided environmental degradation. In Pindyck (2002) the continuous time model of environmental policy adopted in Pindyck (2000) is extended and generalized by focusing on how irreversibilities and uncertainties interact in terms of timing of policy adoption.

In a different framework and with a different focus Bentolila and Bertola (1990) have analyzed the implications of labor adjustment costs - linear hiring costs per new employees and linear firing costs per dismissed workers - on risk-neutral firms' employment policy under stochastic product demand which follows a geometric Brownian motion process. Their results suggest that highly regulated labor markets constrain the flexibility of firms' employment policies because hiring a worker is a risky proposition and the degree of uncertainty about the future is an important parameter in terms of firms' decision making.

In stochastic harvesting and forest rotation models the distinction between price and stocks has not been usually done, but studies have mainly focused on the impact of stochastic forest stand value on the harvesting threshold and the expected rotation length (see e.g. Alvarez (2004), Alvarez and Koskela (2004), Sødal (2002) and Willassen (1998)). The early papers on optimal harvesting under stochastic timber prices typically assume that the underlying timber price evolves according to a geometric Brownian motion (cf. Clarke and Reed (1989), Reed and Clarke (1990), Morck et. al (1989) and Thomson (1992)). Plantinga (1998) is an example of a paper which examines meanreverting prices numerically. See also Gjolberg and Guttormsen (2002). Insley (2002) contrasts the implication of geometric Brownian motion and mean reverting process of timber prices on harvesting decisions in the single rotation framework. The paper by Insley and Rollins (2004) extends this model to an ongoing rotations framework under mean reverting timber prices with the bare land value determined endogenously. They elaborate forest stand value by postulating stochastic timber prices and deterministic wood volume. Their focus is, however, on numerical illustrations associated with the Ontario forest sector. Saphores (2003) has assumed a concave utility function for the resource manager and studies the issue that allows for partial harvests and accounts for the risk of extinction and for biological assets with the size-dependent stochastic growth. He derived a generalized version of the Faustmann formula both for general growth functions and for harvesting cost specifications. 
In this paper we separate the stochastic price and the stochastic stock dynamics and analyze the optimal harvesting policy and its determinants both theoretically and by using explicit numerical illustrations. We derive several new results. First, we characterize the circumstances under which the immediate depletion of the harvested stock is optimal and state a set of weak conditions under which the optimal harvesting policy can be characterized by a single harvesting threshold, below which harvesting is suboptimal. Moreover, and importantly, we demonstrate how the value of the optimal harvesting policy can be decomposed into the monetary value of the current stock and the expected yield accrued from the future harvesting opportunities resulting from the unharvested stock. Since the instantaneous depletion of the entire stock is an admissible harvesting policy the latter part of the presented decomposition of the value can be interpreted as the excess return accrued from following the optimal policy and leaving part of the stock unharvested (hence the excess return generated by the optimal policy can also be interpreted as the value of waiting). Second, we show that both the value of the optimal harvesting policy and the value of the associated single harvesting opportunity (an optimal depletion policy) can be expressed in a separable form where only the current price and the expected per capita growth of the price process affect the threshold while under risk neutrality the volatility of price dynamics will have no effect. Third, and naturally, the optimal harvesting threshold under stochastic price and stock dynamics exceeds the threshold characterizing the optimal policy in the deterministic case. This means that uncertainty will make waiting valuable and postpone the rational exercise of harvesting opportunities compared with the deterministic case. Thus, our results are in line with the findings of the modern literature on irreversible investment under uncertainty (cf. Dixit and Pindyck (1994)). Fourth, we show that the value of sequential harvesting opportunity dominates the value of the associated optimal depletion problem and that the optimal harvesting threshold is higher when harvesting can be exercised only once than in the sequential case. This observation is based on the intuitively clear property that the required exercise premium is higher when harvesting can be exercised only once than in the case where it can be repeated later on in the future. Fifth, and interestingly, our results indicate that higher flexibility of admissible harvesting policies does not only increase the value of the optimal harvesting policy but also increases the rate at which the value grows as a function of the harvested stock. Finally, we present explicit numerical illustrations by modelling the stochastic price as a standard geometric Brownian motion and the stock dynamics as a stochastic mean reverting process. Under these assumptions we analyze the relationship between the stock volatility and the optimal harvesting threshold both in the single and in the sequential harvesting cases. We also study the impact of stock dynamics volatility on the value of the optimal harvesting policy and the optimal depletion policy.

We proceed as follows. In section 2 we present a framework to study the harvesting problem when both price and resource stock are stochastic and demonstrate several new analytical results. Section 3 illustrates our theoretical findings explicitly through numerical calculations. Finally, there is a concluding section.

\section{The Harvesting Problem}

In this section we characterize the optimal harvesting problem both in terms of harvesting size and timing in a general framework under stochastic price and stochastic stock dynamics. We proceed as follows: First, we specify the underlying resource stock and price dynamics. Second, we provide a set of weak conditions under which the optimal harvesting policy can be characterized by a single harvesting threshold. Moreover, we provide alternative characterizations of the value of the optimal 
harvesting policy by decomposing it into the monetary value of the current stock and of the expected yield accrued from the future harvesting opportunities resulting from the unharvested stock. Third, under these conditions we characterize both the value of the optimal sequential harvesting policy and the value of the associated single harvesting opportunity as the separable form in terms of the impact of the current price and the expected per capita growth rate on the harvesting threshold. Finally, we characterize the properties of the optimal harvesting policy and compare the value of the sequential harvesting opportunity both to the value policy of the associated optimal depletion policy and to the deterministic case.

We assume that the stochastic dynamics of the underlying harvested stock are described by the Itô stochastic differential equation

$$
d X_{t}^{Z}=\mu\left(X_{t}^{Z}\right) d t+\eta \sigma\left(X_{t}^{Z}\right) d W_{t}-d Z_{t}, \quad X_{0}^{Z}=x
$$

where $\eta \geq 0$ is an exogenously given constant multiplier and $W_{t}$ is Brownian motion. We assume that the volatility coefficient $\sigma: \mathbb{R}_{+} \mapsto \mathbb{R}_{+}$is positive at all states (i.e. that $\sigma(x)>0$ for all $x \in \mathbb{R}_{+}$) and that both the drift coefficient $\mu: \mathbb{R}_{+} \mapsto \mathbb{R}$ and the volatility coefficient $\sigma(x)$ are sufficiently smooth (at least continuous) mappings guaranteeing the existence of a solution for (2.1). We call an irreversible harvesting policy $Z$ admissible if it is non-negative, non-decreasing, right-continuous, and $\left\{\mathcal{F}_{t}\right\}$-adapted, and denote the set of admissible harvesting policies as $\Lambda$. We denote as $X_{t}$ the controlled stochastic resource stock dynamics in the absence of harvesting (i.e. when $Z \equiv 0$ ) and assume that the upper boundary $\infty$ is natural for $X_{t}$. We also assume that the lower boundary 0 is either natural, exit, or regular for $X_{t}$. In case it is regular, we assume that is killing (see Borodin and Salminen (2002), pp. 14-20, for a thorough characterization of the boundary behavior of linear diffusions).

Having characterized the stochastic dynamics of the harvested stock modelling the stochastically fluctuating renewable resource, we now assume that the price of a harvested unit of stock evolves on the state-space $\mathbb{R}_{+}$according to the diffusion characterized by the following stochastic differential equation

$$
d p_{t}=\alpha p_{t} d t+\beta\left(p_{t}\right) p_{t} d \tilde{W}_{t}, \quad p_{0}=p \in \mathbb{R}_{+}
$$

where the expected per capita growth rate $\alpha \in \mathbb{R}_{+}$is an exogenously given constant, $\tilde{W}_{t}$ is Brownian motion which is assumed to be independent of $W_{t}$ driving the stochastic dynamics in (2.1), and $\beta: \mathbb{R}_{+} \mapsto \mathbb{R}_{+}$is a sufficiently smooth mapping satisfying the standard Novikov condition (cf. Øksendal (2003), p. 162). This assumption implies that, although the volatility coefficient of the diffusion characterizing the underlying price dynamics may be non-linear, the price is always expected to grow at an exponential rate, that is, $\mathbf{E}\left[p_{t}\right]=p e^{\alpha t}$. We also assume that both 0 and $\infty$ are natural boundaries for the price process $p_{t}$ and, therefore, that even though $p_{t}$ may tend towards the boundaries of its state space it never attains these boundaries in finite time.

Given these assumptions, we now investigate the optimal sequential harvesting problem (a singular stochastic control problem)

$$
V(x, p)=\sup _{Z \in \Lambda} \mathbf{E}_{(x, p)} \int_{0}^{\tau_{0}^{Z}} e^{-r s} p_{s} d Z_{s},
$$

where $\tau_{0}^{Z}=\inf \left\{t \geq 0: X_{t}^{Z} \leq 0\right\}$ denotes the first date at which the harvested stock is depleted and becomes extinct. For the sake of comparison, we also consider the associated optimal depletion 
problem

$$
J(x, p)=\sup _{\tau<\tau_{0}} \mathbf{E}_{(x, p)}\left[e^{-r \tau} p_{\tau} X_{\tau}\right],
$$

where $\tau_{0}=\inf \left\{t \geq 0: X_{t} \leq 0\right\}$ denotes the first date at which the harvested stock vanishes. It is worth noticing that problem (2.4) can be interpreted as the determination of the date at which a single harvesting opportunity should be exercised. Since this depletion policy belongs into the class of admissible harvesting policies $\Lambda$, we observe that the value $J(x, p)$ can always be attained in the sequential harvesting case while the opposite result is naturally not true. Before stating our first result on the optimal harvesting policies and their values, we first define the mapping $\pi: \mathbb{R}_{+} \mapsto \mathbb{R}$ measuring the growth rate of the expected net present value of the harvested stock as

$$
\pi(x)=\mu(x)-(r-\alpha) x .
$$

We will assume throughout this study that the expected cumulative net present value of the flow $\pi(x)$ from the present up to an arbitrarily distant future exists and is finite. We can now establish the following.

Lemma 2.1. Assume that the expected net present value of the harvested stock is non-increasing. Then, the optimal policy is to instantaneously deplete the entire stock. Put formally, if $\mu(x)+\alpha x \leq$ $r x$ for all $x \in \mathbb{R}_{+}$, then $V(x, p)=J(x, p)=p x, Z_{0}=x, \tau_{0}^{Z}=0$, and $\tau=0$.

Proof. See Appendix A.

Lemma 2.1 characterizes those circumstances under which the immediate depletion of the harvested stock is optimal. As intuitively is clear, postponing the harvesting decision into the future is suboptimal whenever the expected rate of return associated with the deferral of the irreversible decision falls short of its opportunity cost at all states. Consequently, this means that in the case of Lemma 2.1 the excess return associated with the deferral of the harvesting decision further into the future is zero.

Having considered the case where the instantaneous depletion of the harvested stock is optimal, we now proceed to analyze the more complex cases where waiting is valuable and, therefore, where the immediate depletion of a renewable resource is not optimal. In order to accomplish that task, we now assume that the discount rate dominates the percentage growth rate of the price, that is, that $r>\alpha$, and denote as $\psi_{\eta}(x)$ the increasing fundamental solution of the ordinary differential equation (cf. Borodin and Salminen (2002), pp. 17-19)

$$
\frac{1}{2} \eta^{2} \sigma^{2}(x) u^{\prime \prime}(x)+\mu(x) u^{\prime}(x)-(r-\alpha) u(x)=0 .
$$

Our main result characterizing the optimal sequential harvesting policy for a broad class of processes modelling the stochastic dynamics of the underlying harvested stock is now summarized in the following.

Theorem 2.2. Assume that the growth rate of the expected net present value of the harvested stock satisfies the conditions $\lim _{x \rightarrow \infty} \pi(x)<0$ and that

(i) if 0 is unattainable for $X_{t}$ then there is a unique threshold $\hat{x} \in(0, \infty)$ such that $\pi(x)$ is increasing on $(0, \hat{x})$, decreasing on $(\hat{x}, \infty)$, and $\lim _{x \downarrow 0} \mu(x) \geq 0$; 
(ii) if 0 is attainable for $X_{t}$ then there is a unique threshold $\hat{x} \in[0, \infty)$ such that $\pi(x)$ is increasing on $(0, \hat{x})$, decreasing on $(\hat{x}, \infty)$ and $\lim _{x \downarrow 0} \mu(x)>0$.

Then, the value of the optimal harvesting policy reads as

$$
V(x, p)= \begin{cases}p x+\frac{p \pi\left(x_{\eta}^{*}\right)}{r-\alpha} & x \geq x_{\eta}^{*} \\ \frac{p \psi_{\eta}(x)}{\psi_{\eta}^{\prime}\left(x_{\eta}^{*}\right)} & x<x_{\eta}^{*}\end{cases}
$$

where the optimal harvesting threshold $x_{\eta}^{*}=\operatorname{argmin}\left\{\psi_{\eta}^{\prime}(x)\right\}>\hat{x}$ is the unique root of the ordinary necessary first order optimality condition $\psi_{\eta}^{\prime \prime}\left(x_{\eta}^{*}\right)=0$. Moreover, $V_{x}(x, p) \geq p$ and $V_{x x}(x, p) \leq 0$ for all $(x, p) \in \mathbb{R}_{+}^{2}$.

Proof. See Appendix B.

Theorem 2.2 states a set of weak conditions under which the optimal harvesting policy can be characterized by a single harvesting threshold below which harvesting is always suboptimal. Especially, Theorem 2.2 proves that the value of the optimal policy can be expressed in the separable form

$$
V(x, p)=p \sup _{Z \in \Lambda} \mathbf{E}_{x} \int_{0}^{\tau_{0}^{Z}} e^{-(r-\alpha) s} d Z_{s} .
$$

Thus, we observe that in the present example only the current price and its expected per capita growth rate affect the optimal harvesting threshold and its value while the volatility of the price dynamics has no effect on the optimal policy. This observation is naturally based on the assumed risk neutrality of the resource manager. It is also worth noticing that Theorem 2.2 proves that the optimal harvesting threshold $x_{\eta}^{*}$ exceeds the threshold $\hat{x}$ at which the growth rate of the expected net present value of the harvested stock is at its maximum. Since $\hat{x}$ is the threshold at which the harvesting opportunity is exercised in the deterministic case, we find that in the present case the stochasticity of the dynamics of the harvested stock makes waiting valuable and thus postpones the rational exercise in comparison with the deterministic case.

It is worth noticing that the conditions of Theorem 2.2 are relatively weak, since no strong concavity properties are required for the validity of the conclusions of our theorem. However, it is clear that most models subject to mean reversion (for example, models subject to pure compensation; cf. Clark (1976), section 1.1) satisfy the conditions of Theorem 2.2. This result is established in the following.

Corollary 2.3. Assume that the drift coefficient $\mu(x)$ is continuously differentiable, strictly concave, and satisfies the boundary conditions $\mu(0)=0$ and $\lim _{x \downarrow 0} \mu^{\prime}(x)>r-\alpha>\lim _{x \rightarrow \infty} \mu^{\prime}(x)$. Then the conditions of Theorem 2.2 are satisfied and the value of the optimal harvesting policy reads as in (2.6).

Proof. Under assumptions of our corollary $\pi(x)$ is continuously differentiable, strictly concave, and satisfies the boundary conditions $\pi(0)=0$ and $\lim _{x \downarrow 0} \pi^{\prime}(x)>0>\lim _{x \rightarrow \infty} \pi^{\prime}(x)$. Thus, the continuity and monotonicity of the derivative $\pi^{\prime}(x)$ implies that the mapping $\pi(x)$ attains a unique global maximum at $\hat{x} \in \mathbb{R}_{+}$. Moreover, for all $x \geq y>\hat{x}$ it holds

$$
\pi(x) \leq \pi(y)+\pi^{\prime}(y)(x-y)
$$

proving that $\pi(x) \downarrow-\infty$ as $x \rightarrow \infty$. Thus, the conditions of Theorem 2.2 are satisfied and the alleged result follows. 
Corollary 2.3 states a set of regularity conditions under which the conditions of Theorem 2.2 are satisfied and, therefore, under which the optimal harvesting threshold can be characterized by a single harvesting threshold at which harvesting should be irreversibly initiated. It is worth noticing that since the optimal threshold is attained on the set where the expected growth rate of the net present value of the harvested stock is decreasing we have the inequality $\mu^{\prime}\left(x_{\eta}^{*}\right)+\alpha \leq r$ so that at the optimum the marginal rate of return from a harvested unit has to fall short its opportunity cost. A second important implication of Theorem 2.2 presenting the value of the optimal harvesting policy in terms of the value of instantaneous depletion of the harvested stock and the value of the future harvesting potential is now summarized in the following.

Corollary 2.4. Assume that the conditions of Theorem 2.2 are satisfied. Then, the value of the optimal harvesting policy can be re-expressed on the set $\left(0, x_{\eta}^{*}\right)$ where harvesting is suboptimal as $V(x, p)=p x+p K(x)$, where

$$
K(x)=\left(R_{r-\alpha} \pi\right)(x)-\frac{\left(R_{r-\alpha} \pi\right)^{\prime}\left(x_{\eta}^{*}\right)}{\psi_{\eta}^{\prime}\left(x_{\eta}^{*}\right)} \psi_{\eta}(x),
$$

measures the expected cumulative stock of the future harvesting opportunities and

$$
\left(R_{r-\alpha} \pi\right)(x)=\mathbf{E}_{x} \int_{0}^{\tau_{0}} e^{-(r-\alpha) s} \pi\left(X_{s}\right) d s
$$

describes the expected cumulative present value of the flow $\pi(x)$ from the present up to a potentially infinite future.

Proof. See Appendix C.

Corollary 2.4 presents an alternative characterization of the value of the optimal harvesting policy compared with (2.6) in Theorem 2.2. According to this representation the value can be expressed in terms of the monetary value of the current stock and the expected yield accrued from the future harvesting opportunities resulting from leaving a part of the stock unharvested. Hence, the formulation of the value of the optimal harvesting policy presented in Corollary 2.4 can be interpreted as an intertemporal decomposition of the values of the harvesting opportunities available to the harvester. Moreover, since $p x$ measures the monetary value of the current stock, we find that the term $p K(x)$ can be interpreted as the excess monetary return accrued by following the optimal policy and leaving part of the stock unharvested. A third important implication of Theorem 2.2 characterizing the marginal value of the harvesting opportunity (and, therefore, Tobin's marginal q; for an excellent survey of the classical q-theory of investment, see Abel 1990 and Caballero 1999) is now stated in our next corollary.

Corollary 2.5. Assume that the conditions of Theorem 2.2 are satisfied. Then, the marginal value of the optimal harvesting policy can be re-expressed as

$$
V_{x}(x, p)=p \psi_{\eta}^{\prime}(x) \inf _{y \geq x}\left[\frac{1}{\psi_{\eta}^{\prime}(y)}\right]=p+p\left(R_{r-\alpha} \pi\right)^{\prime}(x)-p \psi_{\eta}^{\prime}(x) \inf _{y \geq x}\left[\frac{\left(R_{r-\alpha} \pi\right)^{\prime}(y)}{\psi_{\eta}^{\prime}(y)}\right] .
$$

Especially, if $\mu(x)$ and $\sigma(x)$ are continuously differentiable with Lipschitz-continuous derivatives and $\sigma^{\prime}(x)$ is bounded, then (2.7) can be re-expressed as

$$
V_{x}(x, p)=p \sup _{\tau<\hat{\tau}_{0}} \mathbf{E}_{x}\left[e^{\int_{0}^{\tau} \pi^{\prime}\left(\hat{X}_{s}\right) d s}\right]
$$


where the process $\hat{X}_{t}$ evolves according to the stochastic dynamics described by the stochastic differential equation

$$
d \hat{X}_{t}=\left(\mu\left(\hat{X}_{t}\right)+\sigma^{\prime}\left(\hat{X}_{t}\right) \sigma\left(\hat{X}_{t}\right)\right) d t+\sigma\left(\hat{X}_{t}\right) d \hat{W}_{t}, \quad \hat{X}_{0}=x,
$$

and $\hat{\tau}_{0}=\inf \left\{t \geq 0: \hat{X}_{t} \leq 0\right\}$

Proof. See Appendix D.

Corollary 2.5 demonstrates that the marginal value of the optimal harvesting policy can be expressed in terms of an associated non-linear programming problem. Thus, Corollary 2.5 proves that the optimal harvesting policy does not only maximize the expected cumulative present value of the harvesting yield from the present up to a potentially infinite future, but it simultaneously also maximizes the rate at which this value grows (and, therefore, the marginal value of a harvested unit of stock). Interestingly, our results indicate that under a set of sufficient smoothness conditions the marginal value of the harvesting opportunity can be interpreted in terms of an associated optimal timing problem. A fourth interesting implication of the findings of Theorem 2.2, demonstrating how the flexibility of an admissible harvesting policy affects the maximal attainable expected cumulative present value of the future harvesting yields, is now summarized in the following.

Corollary 2.6. Assume that the conditions of our Theorem 2.2 are satisfied. Then the value of the optimal harvesting strategy satisfies the inequality $V(x, p) \geq I_{c}(x, p)$, where

$$
I_{c}(x, p)=\sup _{\nu \in \Gamma} \mathbf{E}_{(x, p)} \sum_{k=1}^{N} e^{-r \tau_{k}}\left[p_{\tau_{k}} X_{\tau_{k}-}^{\nu}-c\right]
$$

denotes the value of the associated stochastic impulse control problem, $c \geq 0$ is a known exogenously given constant measuring the harvesting costs, $\Gamma$ is the set of admissible harvesting strategies characterized by the potentially infinite joint sequence $\nu=\left\{\left(\tau_{k}, \zeta_{k}\right)\right\}_{k=1}^{N}, N \leq \infty$, where $\left\{\tau_{k}\right\}_{k=1}^{N}$ denotes an increasing sequence of harvesting dates and $\left\{\zeta_{k}\right\}_{k=1}^{N}$ denotes a sequence of non-negative harvests exerted at the corresponding harvesting dates, and

$$
X_{t}^{\nu}=x+\int_{0}^{t} \mu\left(X_{s}^{\nu}\right) d s+\int_{0}^{t} \sigma\left(X_{s}^{\nu}\right) d W_{s}-\sum_{j=1}^{K} \zeta_{j}, \quad t \in\left[\tau_{K}, \tau_{K+1}\right) .
$$

Proof. See Appendix E.

Corollary 2.6 demonstrates the intuitively clear result that increased flexibility increases the value of the harvesting opportunity. More precisely, according to Corollary 2.6 the value of the associated discrete impulse control problem (which is known in forest economics as a Faustmannian ongoing rotation problem) is smaller than the value of the considered sequential optimal harvesting problem.

Having presented the main implications of our main theorem characterizing the optimal harvesting policy and its value, we now plan to analyze the comparative static properties of the optimal policy and, especially, the impact of increased volatility in the harvested stock on the optimal policy and its value. Our main conclusion on this topic is now summarized in the following.

Theorem 2.7. Assume that the conditions of Theorem 2.2 are satisfied. Then increased volatility decreases the value $V(x, p)$ and increases the optimal exercise threshold $x_{\eta}^{*}$. More precisely, $\partial V(x, p) / \partial \eta<0$ and $\partial x_{\eta}^{*} / \partial \eta>0$. 
Proof. See Appendix F.

Theorem 2.7 demonstrates that given the conditions of Theorem 2.2 higher volatility in the harvested stock decreases the value of the optimal harvesting policy and postpones the rational exercise of the harvesting opportunity by increasing the optimal harvesting threshold at which the harvesting opportunity should be exercised. The negativity of the sign of the relationship between stock volatility and the value of the harvesting opportunity is a natural implication of the concavity of the value of the optimal policy. The positivity of the sign of the relationship between stock volatility and the optimal harvesting threshold, in turn, follows from the monotonicity of the growth rate of the expected net present value of the harvested stock. More precisely, since increased stock volatility decreases the value of the harvesting opportunity while leaving the monetary value of the current stock unchanged, we find that higher stock volatility decreases the excess return associated to the optimal policy as well. Since this excess return can be expressed on the harvesting region in terms of the growth rate of the expected net present value of the harvested stock and this rate is decreasing on the set where harvesting is optimal we find that increased stock volatility will unambiguously decelerate harvesting by increasing the threshold at which the harvesting opportunity should be irreversibly exercised.

Our main result on the associated optimal depletion problem modelling the valuation of a single harvesting opportunity is now summarized in the following.

Theorem 2.8. Assume that there is a threshold $x_{0} \in \mathbb{R}_{+}$such that $\pi(x) \gtreqless 0$ whenever $x \lesseqgtr x_{0}$. Then, the value of the optimal harvesting policy reads as

$$
J(x, p)=p \psi_{\eta}(x) \sup _{y \geq x}\left[\frac{y}{\psi_{\eta}(y)}\right]= \begin{cases}p x & x \geq \tilde{x}_{\eta} \\ p \frac{\psi_{\eta}(x)}{\psi_{\eta}^{\prime}\left(\tilde{x}_{\eta}\right)} & x<\tilde{x}_{\eta},\end{cases}
$$

where the optimal harvesting threshold $\tilde{x}_{\eta}>x_{0}$ is the unique root of the first order optimality condition $\psi_{\eta}\left(\tilde{x}_{\eta}\right)=\tilde{x}_{\eta} \psi_{\eta}^{\prime}\left(\tilde{x}_{\eta}\right)$. Moreover, the value of the optimal harvesting policy can be reexpressed as $J(x, p)=p x+p L(x)$, where

$$
L(x)=\left(R_{r-\alpha} \pi\right)(x)-\psi_{\eta}(x) \inf _{y \geq x}\left[\frac{\left(R_{r-\alpha} \pi\right)(y)}{\psi_{\eta}(y)}\right]
$$

measures the early exercise premium.

Proof. See Appendix G.

Theorem 2.8 states a set of weak conditions under which the optimal depletion policy can be characterized in terms of a single harvesting threshold at which the considered single harvesting opportunity should be irreversibly exercised. As in the case of Theorem 2.2, we again find that the growth rate of the expected net present value of the harvested stock is the principal determinant of the optimal harvesting policy and its value. Along the lines of Theorem 2.2, Theorem 2.8 proves that the value of the optimal depletion policy can be expressed in the separable form

$$
J(x, p)=p \sup _{\tau} \mathbf{E}_{x}\left[e^{-(r-\alpha) \tau} X_{\tau}\right] .
$$

Theorem 2.8 also states a decomposition of the value into a term capturing the monetary value of the current stock and the value accrued from postponing the harvesting decision into the future. 
Interestingly, according to this decomposition the excess return $p L(x)$ accrued from following the optimal policy reads in the continuation region $\left(0, \tilde{x}_{\eta}\right)$ where harvesting is suboptimal as

$$
L(x)=\left(R_{r-\alpha} \pi\right)(x)-\psi_{\eta}(x) \frac{\left(R_{r-\alpha} \pi\right)^{\prime}\left(\tilde{x}_{\eta}\right)}{\psi_{\eta}^{\prime}\left(\tilde{x}_{\eta}\right)} .
$$

This expression resembles the excess return associated to the continuous singular harvesting strategy considered in Theorem 2.2. However, it does not coincide with that value as is established in our following theorem characterizing the relationship between the value of the sequential and the single harvesting opportunity.

Theorem 2.9. Assume that the conditions of Theorem 2.2 are satisfied. Then, $\tilde{x}_{\eta}>x_{\eta}^{*}, V(x, p) \geq$ $J(x, p), V_{x}(x, p) \geq J_{x}(x, p), V_{p}(x, p) \geq J_{p}(x, p)$, and $V_{x p}(x, p) \geq J_{x p}(x, p)$ for all $(p, x) \in \mathbb{R}_{+}^{2}$.

Proof. See Appendix H.

Theorem 2.9 proves that the value of the sequential harvesting opportunity dominates the value of the associated optimal depletion problem. This result is intuitively clear, since the instantaneous depletion of the harvested stock is an admissible policy in the sequential case as well. It is the ability to leave part of the stock unharvested and in this way postpone the depletion of the stock into the future which creates the excess return capturing the value of the future harvesting opportunities and, therefore, measuring the value of waiting. In accordance with this observation, Theorem 2.9 also proves that the optimal harvesting threshold is higher in the case where the harvesting can be exercised only once than in the sequential case. Interestingly, Theorem 2.9 also demonstrates that increased flexibility of the class of admissible policies does not only increase the value of the optimal policy, it also increases the rate at which this value increases (cf. Alvarez and Virtanen (2004)).

\section{Illustration}

In section 2 we demonstrated several new theoretical results on the general harvesting problem in the presence of both stochastic timber price and harvested stock dynamics in the case when harvesting can be exercised only once and in the sequential case when the harvesting opportunity can be repeated later on in the future. We now illustrate these results explicitly by characterizing the underlying stochastic price dynamics as a standard geometric Brownian motion and the stock dynamics as a stochastic mean reverting process. We elaborate the relationship between stock volatility and the optimal harvesting threshold both in the single and in the sequential harvesting cases and show how higher volatility raises the optimal harvesting threshold. Moreover, we illustrate the impact of the volatility of stock dynamics on the value of the optimal sequential harvesting policy and on the value of the optimal depletion policy. Higher volatility decreases the value of sequential harvesting policy while its effect is ambiguous on the value of optimal depletion policy.

In order to illustrate the results of our previous section explicitly we now assume that the unit price $p_{t}$ evolves according to a standard geometric Brownian motion and, therefore, that $\beta(p)=\beta p$, where $\beta>0$ is an exogenously given constant. In order to introduce mean reverting stock dynamics, we assume that $\mu(x)=\mu x(1-\gamma x)$ and that $\sigma(x)=\sigma x$, where $\mu, \gamma, \sigma \in \mathbb{R}_{+}$are exogenously determined constants.

It is now clear that if $\mu \leq r-\alpha$ then the conditions of Lemma 2.1 are satisfied and the instantaneous depletion of the stock is optimal. However, if $\mu>r-\alpha$ then the conditions of part 
(i) of our Theorem 2.2 is satisfied since 0 is a natural boundary for the considered mean reverting diffusion and $\pi^{\prime}(x)=\mu(1-2 \gamma x)-(r-\alpha) \gtreqless 0$ when $x \gtreqless(\mu-r+\alpha) /(2 \gamma)$. In this case,

$$
\psi_{\sigma}(x)=x^{\theta_{\sigma}} M\left(\theta_{\sigma}, 2 \theta_{\sigma}+\frac{2 \mu}{\sigma^{2}}, \frac{2 \mu \gamma}{\sigma^{2}} x\right),
$$

where

$$
\theta_{\sigma}=\frac{1}{2}-\frac{\mu}{\sigma^{2}}+\sqrt{\left(\frac{1}{2}-\frac{\mu}{\sigma^{2}}\right)^{2}+\frac{2(r-\alpha)}{\sigma^{2}}} \in(0,1)
$$

denotes the positive root of the characteristic equation $\sigma^{2} a(a-1) / 2+\mu a-(r-\alpha)=0$, and $M$ denotes the confluent hypergeometric function (cf. Abramowitz and Stegun (1968), pp. 555-566).

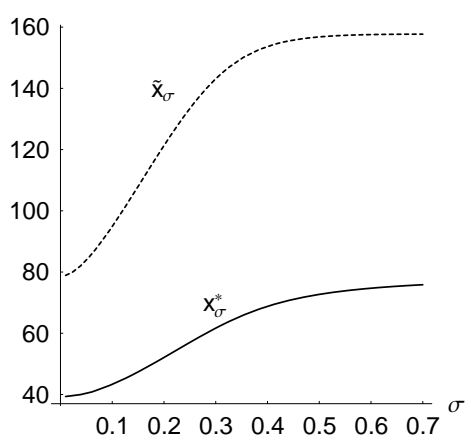

Figure 1: The optimal harvesting thresholds

Figure 1 describes the optimal harvesting thresholds as a function of the resource stock dynamics volatility. Naturally, higher volatility postpones the rational exercise of the optimal harvesting opportunity both in the sequential case and in the case where harvesting will be exercised only once. According to Figure 2 higher volatility decreases the value of the optimal sequential harvesting policy due to the concavity of the value of optimal policy. Figure 3 describes the relationship between volatility and the value of the optimal depletion policy. Now the relationship is ambiguous due to the fact that the optimal depletion threshold is attained on the region where the value function is convex.

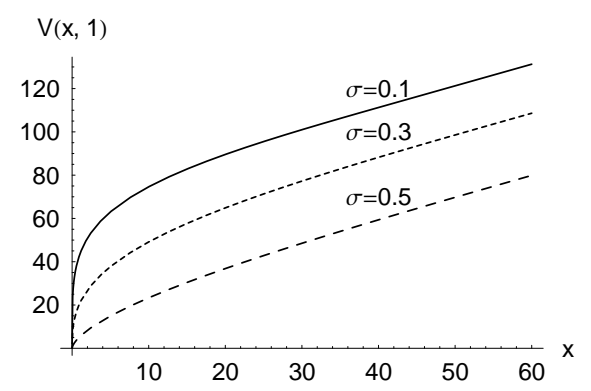

Figure 2: The Impact of Increased Volatility on the Value $V(x, 1)$ 


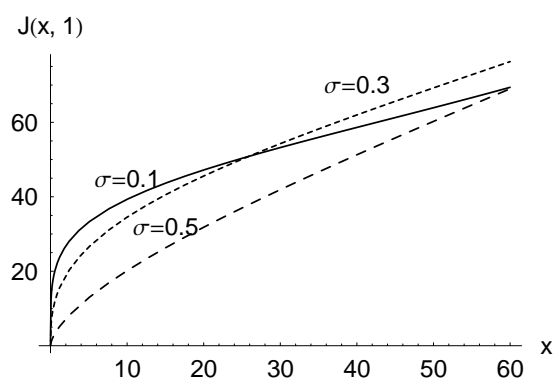

Figure 3: The Impact of Increased Volatility on the Value $J(x, 1)$

\section{Conclusions}

In this paper we have analyzed optimal harvesting policy and its determinants both theoretically and by using explicit numerical illustrations in a general framework where both price and stock dynamics are stochastic and resource manager is risk neutral. We have derived several new results. First, we specified the circumstances under which the immediate depletion of the harvested stock is optimal and stated a set of weak conditions under which the optimal harvesting policy can be characterized by a single harvesting threshold. Second, we have shown that both the value of the optimal harvesting policy and the value of the associated single harvesting opportunity can be expressed as a separable form in such a way that only the current price and the expected per capita growth rate affect the threshold while under risk neutrality the volatility of price dynamics will have no effect. Moreover, we demonstrated how the value of the optimal harvesting policy can be decomposed into the monetary value of the current stock and of the expected yield accrued from the future harvesting opportunities resulting from the unharvested stock. Third, the optimal harvesting threshold under stochastic price and stock dynamics exceeds the threshold of the deterministic case meaning that stochasticity makes waiting valuable and postpones the rational exercise compared with the deterministic case. Fourth, we demonstrated that the value of sequential harvesting opportunity dominates the value of the associated optimal depletion problem and that the optimal harvesting threshold is higher in the case where harvesting can be exercised only once than in the sequential case. Fifth, and interestingly, our findings indicate that higher flexibility of admissible policies does not only increase the value of optimal policy but also increases the rate at which the value increases. Finally, we used explicit numerical illustrations by specifying a standard geometric Brownian motion for stochastic price and a stochastic mean reverting process for stock dynamics to elaborate the relationship between stock volatility and optimal harvesting threshold both in the single and sequential harvesting cases and the impact of volatility on the value of optimal harvesting policy and optimal depletion policy. The optimal harvesting thresholds depend positively on the volatility of stock dynamics both in the sequential case and in the case where harvesting will be exercised only once. Moreover, higher volatility increases the value of the sequential harvesting policy while the relationship between volatility and the value of the optimal depletion policy is ambiguous.

Even though the preset study analyzes a relatively general class of harvesting problem, there are two potentially interesting directions towards which the analysis could be extended. First, it is not clear how relaxing the assumed geometric nature of the local expected growth of the underlying price dynamics affects the optimal harvesting decision and its value. Second, given the length of the considered planning horizon, assuming constant discounting overlooks the potentially significant role of 
interest rate variability and, especially, of interest rate uncertainty. Unfortunately, the introduction of such generalizations is out of the scope of the present study and, therefore, left for future research.

Acknowledgements: The research of Luis H. R. Alvarez has been supported by the Foundation for the Promotion of the Actuarial Profession and the Finnish Insurance Society. Erkki Koskela thanks the Yrjö Jahnsson Foundation for financial support. Both authors acknowledge the financial support from the Research Unit of Economic Structures and Growth (RUESG) at the University of Helsinki. 


\section{References}

[1] Abel, A. B. Consumption and investment, 1990, in Friedman, F. and Hahn, F. (eds), Handbook of Monetary economics, vol II, 725-778, North-Holland, New York.

[2] Abramowitz, M. and Stegun, I. A., eds., Handbook of Mathematical Functions, 1968, Dover Publications, New York.

[3] Alvarez, L. H. R. Singular stochastic control, linear diffusions, and optimal stopping: A class of solvable problems, 2001, SIAM Journal on Control and Optimization, 39, 1697 $-1710$.

[4] Alvarez, L. H. R. A class of solvable impulse control problems, 2004, Applied Mathematics E Optimization, 49, 265-295.

[5] Alvarez, L. H. R. and Koskela, E., Taxation and rotation age under stochastic forest stand value, 2004, Department of Economics, University of Helsinki, Discussion papers, No 604 .

[6] Alvarez, L. H. R. and Virtanen, J. A Class of solvable stochastic dividend optimization problems: On the general impact of flexibility on valuation, 2004, Turku School of Economics and Business Administration, Discussion Paper Series, nr 1/2004.

[7] Bentolila, S. and Bertola, G., Firing costs and labour demand: How bad is Eurosclerosis?, 1990, Review of Economic Studies, 57, 381-402.

[8] Björk, T. Arbitrage Theory in Continuous Time, 1998, Oxford University Press.

[9] Borodin, A. and Salminen, P. Handbook on Brownian Motion - Facts and Formulae, 2nd edition, 2002, Birkhauser, Basel.

[10] Caballero, R. J. Aggregate Investment, in Handbook of Macroeconomics, Vol. 1B, Taylor, J.P. and Woodford, M. (eds), 813-862.

[11] Clark, C. W. Mathematical Bioeconomics: The Optimal Management of Renewable Resources, 1976, Wiley, New York.

[12] Clarke, H. R. and Reed, W. J., The tree-cutting problem in a stochastic environment, 1989, Journal of Economic Dynamics and Control, 13, 569-595.

[13] Dixit, A. K. and Pindyck, R. S. Investment under Uncertainty, 1994, Princeton UP, Princeton.

[14] Gjolberg, O. and Guttormsen, A. G. Real options in the forest: what if prices are meanreverting?, 2002, Forest Policy and Economics, 4, 13-20.

[15] Harrison, J. M. Brownian Motion and Stochastic Flow Systems, 1985, Wiley, New York.

[16] Insley, M. A real options approach to the valuation of a forestry investment, 2002, Journal of Environmental Economics and Management, 44, 471-492.

[17] Insley, M.C. and Rollins, K. On estimating the costs of regulations limiting flexibility in timber harvest decisions: A multi-rotational real options model, mimeo, March 2004. 
[18] Morck, R. and Schwartz, E. and Stangeland, D. The valuation of forestry resources under stochastic prices and inventories, 1989, Journal of Financial and Quantitative Analysis, 24, 473-487.

[19] Øksendal, B. Stochastic Differential Equations: An Introduction with Applications, (Sixth Edition) 2003, Springer, Berlin.

[20] Pindyck, R. S. Uncertainty in the theory of renewable resource markets, 1984, Review of Economic Studies, 51, 289-303.

[21] Pindyck, R. S. Irreversibilities and the timing of environmental policy, 2000, Resource and Energy Economics, 22, 233-259.

[22] Pindyck, R. S. Optimal timing problems in environmental economics, 2002, Journal of Economic Dynamics and Control, 26, 1677-1697.

[23] Plantinga, A. J. The optimal timber rotation: an option value approach, 1998, Forest Science, 44, 192-202.

[24] Reed, W. J. and Clarke, H. R., Harvest decisions and asset valuations for biological resources exhibiting size-dependent stochastic growth, 1990, International Economic Review, 31, 147-169.

[25] Saphores, J.-D. Harvesting a renewable resource under uncertainty, 2003, Journal of Economic Dynamics and Control, 28, 509-529.

[26] Sødal, S. The stochastic rotation problem: A comment, 2002, Journal of Economic Dynamics and Control, 26, 509-515.

[27] Thomson, T. A. Optimal forest rotation when stumpage prices follow a diffusion process, 1992, Land Economics, 68, 329-342.

[28] Willassen, Y. The stochastic rotation problem: A generalization of Faustmann's formula to stochastic forest growth, 1998, Journal of Economic Dynamics and Control, 22, 573596. 


\section{A Proof of Lemma 2.1}

Proof. In order to establish the validity of the alleged result, we first present the following lemma stating a set of sufficient conditions needed for the verification of the optimality of a proposed admissible sequential harvesting strategy.

Lemma A.1. Assume that there is a twice continuously differentiable mapping $H: \mathbb{R}_{+}^{2} \mapsto \mathbb{R}_{+}$ satisfying the conditions

(i) $H_{x}(x, p) \geq p$ for all $(x, p) \in \mathbb{R}_{+}^{2}$,

(ii) $\left(\mathcal{G}_{\eta} H\right)(x, p) \leq r H(x, p)$ for all $(x, p) \in \mathbb{R}_{+}^{2}$, where

$$
\mathcal{G}_{\eta}=\frac{1}{2} \sigma^{2}(x) \frac{\partial^{2}}{\partial x^{2}}+\frac{1}{2} \beta^{2}(p) p^{2} \frac{\partial^{2}}{\partial p^{2}}+\mu(x) \frac{\partial}{\partial x}+\alpha p \frac{\partial}{\partial p}
$$

denotes the differential operator associated with the two-dimensional diffusion $\left(X_{t}, p_{t}\right)$.

Then, $H(x, p) \geq V(x, p)$ for all $(x, p) \in \mathbb{R}_{+}^{2}$.

Proof. Assume that a mapping $H(x, p)$ satisfies the conditions of our Lemma. Then, the generalized Itô theorem implies that

$$
\begin{array}{r}
\mathbf{E}_{(x, p)}\left[e^{-r T_{N}} H\left(X_{T_{N}}^{Z}, p_{T_{N}}\right)\right]=H(x, p)+\mathbf{E}_{(x, p)} \int_{0}^{T_{N}} e^{-r s}\left(\left(\mathcal{G}_{\eta} H\right)\left(X_{s}^{Z}, p_{s}\right)-r H\left(X_{s}^{Z}, p_{s}\right)\right) d s \\
-\mathbf{E}_{(x, p)} \int_{0}^{T_{N}} e^{-r s} H_{x}\left(X_{s}^{Z}, p_{s}\right) d Z_{s}^{c}+\sum_{s \leq T_{N}} e^{-r s}\left(H\left(X_{s-}^{Z}, p_{s}\right)-H\left(X_{s}^{Z}, p_{s}\right)\right),
\end{array}
$$

where $T_{N}=\inf \left\{t \geq 0: X_{t}^{Z} \notin\left(N^{-1}, N\right)\right\} \wedge \inf \left\{t \geq 0: p_{t} \notin\left(N^{-1}, N\right)\right\} \wedge N \wedge \tau_{0}^{Z}$ is an almost surely finite stopping time converging towards $\tau_{0}^{Z}$ as $N \rightarrow \infty$ and $Z_{t}^{c}$ denotes the continuous part of the admissible harvesting policy $Z_{t}$. The continuous differentiability of $H(x, p)$, the mean value theorem, and inequality (i) implies that $H\left(X_{s-}^{Z}, p_{s}\right)-H\left(X_{s}^{Z}, p_{s}\right) \leq-p_{s} \Delta Z_{s}$, where $\Delta Z_{s}$ denotes the jump part of the admissible harvesting policy. Combining this observation with the non-negativity of the value $H(x, p)$ and inequality (ii) finally implies that for all $(x, p) \in \mathbb{R}_{+}^{2}$ and all admissible harvesting policies it holds

$$
H(x, p) \geq \mathbf{E}_{(x, p)} \int_{0}^{T_{N}} e^{-r s} p_{s} d Z_{s}
$$

Letting now $N \uparrow \infty$ and applying the Fatou theorem then yields

$$
H(x, p) \geq \mathbf{E}_{(x, p)} \int_{0}^{\tau_{0}^{Z}} e^{-r s} p_{s} d Z_{s}
$$

Since this inequality is valid for all admissible harvesting policies, it is valid for the optimal as well and, therefore we find that $H(x, p) \geq V(x, p)$.

Given the proof of the verification Lemma A.1 we notice that the twice continuously differentiable mapping $H(x, p)=p x$ satisfies the condition $H_{x}(x, p)=p$ and $\left(\mathcal{G}_{\eta} H\right)(x, p)-r H(x, p)=$ $p \pi(x) \leq 0$ for all $(x, p) \in \mathbb{R}_{+}^{2}$. Consequently, the conditions of Lemma A.1 are satisfied and $V(x, p) \leq p x$. However, since this value can be attained by choosing the admissible harvesting strategy $Z_{0}=x$ (implying that $\tau_{0}^{Z}=0$ ) we find that $V(x, p) \geq p x$ which finally demonstrates that $V(x, p)=p x$. The identity $J(x, p)=p x$ and optimality the of the stopping time $\tau=0$ now follows from the inequality $\left(\mathcal{G}_{\eta} H\right)(x, p)-r H(x, p)=p \pi(x) \leq 0$ for all $(x, p) \in \mathbb{R}_{+}^{2}$. 


\section{B Proof of Theorem 2.2}

Proof. As was established in Alvarez (2004) the uniform integrability of the flow $\pi(x)$ implies that

$$
\frac{1}{2} \eta^{2} \sigma^{2}(x) \frac{\psi_{\eta}^{\prime \prime}(x)}{S_{\eta}^{\prime}(x)}=(r-\alpha) \int_{0}^{x} \psi_{\eta}(y) \pi(y) m_{\eta}^{\prime}(y) d y-\pi(x) \frac{\psi_{\eta}^{\prime}(x)}{S_{\eta}^{\prime}(x)},
$$

where

$$
S_{\eta}^{\prime}(x)=\exp \left(-\int \frac{2 \mu(x) d x}{\eta^{2} \sigma^{2}(x)}\right)
$$

denotes the density of the scale function $S_{\eta}(x)$ and $m_{\eta}^{\prime}(x)=2 /\left(\eta^{2} \sigma^{2}(x) S_{\eta}^{\prime}(x)\right)$ denotes the density of the speed measure $m_{\eta}$ of the underlying diffusion $X_{t}$ (cf. Borodin and Salminen (2002), p. 17). It in now clear that our assumptions imply the existence and uniqueness of a threshold $x_{0}>\hat{x}$ such that $\pi\left(x_{0}\right)=0$. Letting $x \uparrow x_{0}$ in (B.1) now yields

$$
\frac{1}{2} \eta^{2} \sigma^{2}\left(x_{0}\right) \frac{\psi_{\eta}^{\prime \prime}\left(x_{0}\right)}{S_{\eta}^{\prime}\left(x_{0}\right)}=(r-\alpha) \int_{0}^{x_{0}} \psi_{\eta}(y) \pi(y) m_{\eta}^{\prime}(y) d y>0
$$

since our assumptions imply that $\pi(x)>0$ for all $x<x_{0}$. Moreover, as was established in Alvarez (2001) the right hand side of (B.1) is non-increasing (non-decreasing) on the set where the mapping $\pi(x)$ is non-decreasing (non-increasing). Thus, if the conditions of part (ii) our theorem are satisfied then

$$
\lim _{x \downarrow 0}\left[(r-\alpha) \int_{0}^{x} \psi_{\eta}(y) \pi(y) m_{\eta}^{\prime}(y) d y-\pi(x) \frac{\psi_{\eta}^{\prime}(x)}{S_{\eta}^{\prime}(x)}\right]=-\pi(0) \frac{\psi_{\eta}^{\prime}(0)}{S_{\eta}^{\prime}(0)}<0
$$

proving the existence and uniqueness of $x_{\eta}^{*}=\operatorname{argmin}\left\{\psi_{\eta}^{\prime}(x)\right\} \in\left(\hat{x}, x_{0}\right)$ in that case. On the other hand, if the conditions of part (i) are satisfied then (B.1) can be re-expressed as

$$
\frac{1}{2} \eta^{2} \sigma^{2}(x) \frac{\psi_{\eta}^{\prime \prime}(x)}{S_{\eta}^{\prime}(x)}=(r-\alpha) \int_{0}^{x} \psi_{\eta}(y)(\pi(y)-\pi(x)) m_{\eta}^{\prime}(y) d y
$$

which, in turn, implies that $\psi_{\eta}^{\prime \prime}(x)<0$ for all $x \in(0, \hat{x})$ and, therefore, proves the existence and uniqueness of $x_{\eta}^{*}=\operatorname{argmin}\left\{\psi_{\eta}^{\prime}(x)\right\} \in\left(\hat{x}, x_{0}\right)$ in that case as well. Moreover, since $\psi_{\eta}(x)$ satisfies the ordinary second order linear differential equation (2.5) we find by letting $x \rightarrow x_{\eta}^{*}$ that $\mu\left(x_{\eta}^{*}\right) \psi_{\eta}^{\prime}\left(x_{\eta}^{*}\right)=(r-\alpha) \psi_{\eta}\left(x_{\eta}^{*}\right)$.

Denote now the proposed value function as $\bar{V}(x, p)$. Given the observations stated above we immediately find that $\bar{V}(x, p)$ is twice continuously differentiable and satisfies the variational inequality $\bar{V}_{x}(x, p) \geq p$ for all $(x, p) \in \mathbb{R}_{+}^{2}$. Moreover, since $\left(\mathcal{G}_{\eta} \bar{V}\right)(x, p)=r \bar{V}(x, p)$ for all $(p, x) \in \mathbb{R}_{+} \times\left(0, x_{\eta}^{*}\right)$ and $\left(\mathcal{G}_{\eta} \bar{V}\right)(x, p)-r \bar{V}(x, p)=p\left(\pi(x)-\pi\left(x_{\eta}^{*}\right)\right) \leq 0$ for all $(p, x) \in \mathbb{R}_{+} \times\left(x_{\eta}^{*}, \infty\right)$ (since the optimal threshold is attained on the set where $\pi(x)$ is decreasing) we find that the proposed value function satisfies the conditions of our auxiliary lemma A.1 and, therefore, dominates the value of the optimal policy, that is, $\bar{V}(x, p) \geq V(x, p)$. However, since the proposed value can be attained by applying the admissible singular control policy (a local time push; cf. Harrison (1985))

$$
Z_{t}= \begin{cases}\left(x-x_{\eta}^{*}\right)^{+} & t=0 \\ \mathcal{L}\left(t, x_{\eta}^{*}\right) & t>0\end{cases}
$$

where $\mathcal{L}\left(t, x_{\eta}^{*}\right)$ denotes the local time of the process $X_{t}$ at the state $x_{\eta}^{*}$ we find that $V(x, p) \geq \bar{V}(x, p)$ proving that $V(x, p)=\bar{V}(x, p)$. Finally, since $\psi_{\eta}^{\prime \prime}(x)<0$ for all $x \in\left(0, x_{\eta}^{*}\right)$, we find that $V_{x x}(x, p) \leq 0$ for all $(x, p) \in \mathbb{R}_{+}^{2}$. 


\section{Proof of Corollary 2.4}

Proof. Given the assumed uniform integrability of the flow $\pi(x)$ we know that the expected cumulative present value of the flow $\pi(x)$ from the present up to a potentially infinite future can be expressed as

$$
\left(R_{r-\alpha} \pi\right)(x)=B^{-1} \varphi_{\eta}(x) \int_{0}^{x} \psi_{\eta}(y) \pi(y) m_{\eta}^{\prime}(y) d y+B^{-1} \psi_{\eta}(x) \int_{x}^{\infty} \varphi_{\eta}(y) \pi(y) m_{\eta}^{\prime}(y) d y,
$$

where $\varphi_{\eta}(x)$ denotes the decreasing fundamental solution of the ordinary linear second order differential equation (2.5) and $B=\left(\psi_{\eta}^{\prime}(x) \varphi_{\eta}(x)-\psi_{\eta}(x) \varphi_{\eta}^{\prime}(x)\right) / S_{\eta}^{\prime}(x)>0$ denotes the constant Wronskian of the fundamental solutions (cf. Borodin and Salminen (2002), pp. 17-19). Standard differentiation of (C.1) now yields that

$$
\frac{\psi_{\eta}^{\prime}(x)}{S_{\eta}^{\prime}(x)}\left(R_{r-\alpha} \pi\right)(x)-\frac{\left(R_{r-\alpha} \pi\right)^{\prime}(x)}{S_{\eta}^{\prime}(x)} \psi_{\eta}(x)=\int_{0}^{x} \psi_{\eta}(y) \pi(y) m_{\eta}^{\prime}(y) d y .
$$

Combining this observation with the identity

$$
\frac{\psi_{\eta}(x)}{S_{\eta}^{\prime}(x)}-x \frac{\psi_{\eta}^{\prime}(x)}{S_{\eta}^{\prime}(x)}=\int_{0}^{x} \psi_{\eta}(y) \pi(y) m_{\eta}^{\prime}(y) d y
$$

now implies that

$$
\frac{\left(R_{r-\alpha} \pi\right)^{\prime}(x)+1}{\psi_{\eta}^{\prime}(x)}=\frac{\left(R_{r-\alpha} \pi\right)(x)+x}{\psi_{\eta}(x)}
$$

and, therefore, that

$$
\frac{d}{d x}\left[\frac{\left(R_{r-\alpha} \pi\right)(x)+x}{\psi_{\eta}(x)}\right]=0 .
$$

Hence, combining these observations now prove that for all $x \in \mathbb{R}_{+}$it holds that

$$
\frac{\left(R_{r-\alpha} \pi\right)(x)+x}{\psi_{\eta}(x)}=\frac{\left(R_{r-\alpha} \pi\right)\left(x_{\eta}^{*}\right)+x_{\eta}^{*}}{\psi_{\eta}\left(x_{\eta}^{*}\right)}=\frac{\left(R_{r-\alpha} \pi\right)^{\prime}\left(x_{\eta}^{*}\right)+1}{\psi_{\eta}^{\prime}\left(x_{\eta}^{*}\right)}
$$

from which the alleged result follows.

\section{Proof of Corollary 2.5}

Proof. The first representation of the marginal value follows from the inequality $\psi_{\eta}^{\prime \prime}(x) \lesseqgtr 0$ when $x \lesseqgtr x_{\eta}^{*}$. On the other hand, applying (C.1) now yields

$$
\frac{d}{d x}\left[\frac{\left(R_{r-\alpha} \pi\right)^{\prime}(x)}{\psi_{\eta}^{\prime}(x)}\right]=\frac{2 S_{\eta}^{\prime}(x)}{\eta^{2} \sigma^{2}(x)}\left[(r-\alpha) \int_{0}^{x} \psi_{\eta}(y) \pi(y) m_{\eta}^{\prime}(y) d y-\pi(x) \frac{\psi_{\eta}^{\prime}(x)}{S_{\eta}^{\prime}(x)}\right]=\psi_{\eta}^{\prime \prime}(x) .
$$

Hence,

$$
\frac{d}{d x}\left[\frac{\left(R_{r-\alpha} \pi\right)^{\prime}(x)}{\psi_{\eta}^{\prime}(x)}\right] \lesseqgtr 0, \quad x \lesseqgtr x_{\eta}^{*}=\operatorname{argmin}\left\{\frac{\left(R_{r-\alpha} \pi\right)^{\prime}(x)}{\psi_{\eta}^{\prime}(x)}\right\}
$$

from which the latter representation follows. The associated optimal stopping problem can then be derived as in Alvarez (2001). 


\section{E Proof of Corollary 2.6}

Proof. As was established in the proof of Theorem 2.2 the value of the optimal harvesting policy satisfies the conditions (a) $V \in C^{2}\left(\mathbb{R}_{+}^{2}\right)$, (b) $\left(\mathcal{G}_{\eta} V\right)(x, p)-r V(x, p) \leq 0$ for all $(x, p) \in \mathbb{R}_{+}^{2}$, and (c) $V_{x}(x, p) \geq p$ for all $(x, p) \in \mathbb{R}_{+}^{2}$. Standard integration of the inequality (c) over the set $[x, x-\zeta]$ yields $V(x, p)-V(x-\zeta, p) \geq p \zeta \geq p \zeta-c$. Therefore, we find that the value $V(x, p)$ satisfies for all $(x, p) \in \mathbb{R}_{+}^{2}$ the quasi-variational inequality

$$
V(x, p) \geq \sup _{\zeta \in[0, x]}[p \zeta-c+V(x-\zeta, p)]
$$

which finally proves that $V(x, p) \geq I_{c}(x, p)$.

\section{F $\quad$ Proof of Theorem 2.7}

Proof. Denote the value of the optimal harvesting policy defined with respect to the more volatile dynamics characterized by the parameter $\hat{\eta}>\eta$ as $\hat{V}(x, p)$. The concavity and twice continuous differentiability of $V(x, p)$ now implies that for all $(x, p) \in \mathbb{R}_{+}^{2}$ it holds

$$
\left(\mathcal{G}_{\hat{\eta}} V\right)(x, p)-r V(x, p) \leq\left(\left(\mathcal{G}_{\hat{\eta}}-\mathcal{G}_{\eta}\right) V\right)(x, p)=\frac{1}{2} \sigma^{2}(x)\left(\hat{\eta}^{2}-\eta^{2}\right) V_{x x}(x, p) \leq 0 .
$$

Since $V_{x}(x, p) \geq p$ for all $(x, p) \in \mathbb{R}_{+}^{2}$ as well, we find that $V(x, p)$ satisfies the conditions of the verification Lemma A.1 and, therefore, satisfies the inequality $V(x, p) \geq \hat{V}(x, p)$. In order to establish that increased volatility increases the optimal harvesting threshold we observe that on $\left(\max \left(x_{\eta}^{*}, x_{\hat{\eta}}^{*}\right), \infty\right)$ we have $V(x, p)-\hat{V}(x, p)=p\left(\pi\left(x_{\eta}^{*}\right)-\pi\left(x_{\hat{\eta}}^{*}\right)\right) /(r-\alpha) \geq 0$ implying that $x_{\eta}^{*} \leq x_{\hat{\eta}}^{*}$ since the optimal threshold is attained on the set where $\pi(x)$ is decreasing.

\section{G Proof of Theorem 2.8}

Proof. Equation (C.3) implies that

$$
\frac{d}{d x}\left[\frac{x}{\psi_{\eta}(x)}\right]=\frac{S_{\eta}^{\prime}(x)}{\psi_{\eta}^{2}(x)} \int_{0}^{x} \psi_{\eta}(y) \pi(y) m_{\eta}^{\prime}(y) d y .
$$

Thus, we observe that $x / \psi_{\eta}(x)$ is increasing as long as $x \leq x_{0}$ since $\pi(x)>0$ for all $x \in\left(0, x_{0}\right)$. Assume now that $x>k>x_{0}$. Then, the standard mean value theorem for integrals implies that

$$
\int_{0}^{x} \psi_{\eta}(y) \pi(y) m_{\eta}^{\prime}(y) d y=\int_{0}^{k} \psi_{\eta}(y) \pi(y) m_{\eta}^{\prime}(y) d y+\frac{\pi(\xi)}{r-\alpha}\left[\frac{\psi_{\eta}^{\prime}(x)}{S_{\eta}^{\prime}(x)}-\frac{\psi_{\eta}^{\prime}(k)}{S_{\eta}^{\prime}(k)}\right]
$$

and, therefore, that

$$
\lim _{x \rightarrow \infty} \int_{0}^{x} \psi_{\eta}(y) \pi(y) m_{\eta}^{\prime}(y) d y=-\infty
$$

since $\psi_{\eta}^{\prime}(x) / S_{\eta}^{\prime}(x) \rightarrow \infty$ as $x \rightarrow \infty$ and $\pi(\xi)<0$. This proves that $x / \psi_{\eta}(x)$ attains at least one maximum on the set $\left(x_{0}, \infty\right)$. Uniqueness now follows from the inequality

$$
\frac{d}{d x} \int_{0}^{x} \psi_{\eta}(y) \pi(y) m_{\eta}^{\prime}(y) d y=\psi_{\eta}(x) \pi(x) m_{\eta}^{\prime}(x) \gtreqless 0, \quad x \lesseqgtr x_{0} .
$$


Denote now as $\bar{J}(x, p)$ the proposed value function. Since

$$
\bar{J}(x, p)=p \mathbf{E}_{x}\left[e^{-(r-\alpha) \tau_{\tilde{x}_{\eta}}} X_{\tilde{x}_{\eta}}\right],
$$

where $\tilde{x}_{\eta}=\inf \left\{t \geq 0: X_{t} \geq \tilde{x}_{\eta}\right\}$ denotes the potentially optimal harvesting date we immediately find that $J(x, p) \geq \bar{J}(x, p)$. To prove the opposite inequality, we first observe that the proposed value function is continuously differentiable on $\mathbb{R}_{+}^{2}$, twice continuously differentiable outside the harvesting threshold $\tilde{x}_{\eta}$, and satisfies the conditions $\bar{J}_{x x}\left(\tilde{x}_{\eta}+, p\right)=0 \leq \bar{J}_{x x}\left(\tilde{x}_{\eta}-, p\right)=p \psi_{\eta}^{\prime \prime}\left(\tilde{x}_{\eta}\right) / \psi_{\eta}^{\prime}\left(\tilde{x}_{\eta}\right)<\infty$. Moreover, since $\left(\mathcal{G}_{\eta} \bar{J}\right)(x, p)=r \bar{J}(x, p)$ on $\mathbb{R}_{+} \times\left(0, \tilde{x}_{\eta}\right)$ and $\left(\mathcal{G}_{\eta} \bar{J}\right)(x, p)-r \bar{J}(x, p)=p \pi(x) \leq 0$ on $\mathbb{R}_{+} \times\left(\tilde{x}_{\eta}, \infty\right)$ we notice that $\bar{J}(x, p)$ constitutes a $r$-excessive majorant of the payoff $p x$ for the diffusion $\left(X_{t}, p_{t}\right)$ and, therefore, that $J(x, p) \leq \bar{J}(x, p)$ which proves that $J(x, p)=\bar{J}(x, p)$. Finally, the representations of the value function follow from (G.1) and from (C.5).

\section{H Proof of Theorem 2.9}

Proof. Since $x_{\eta}^{*} \in\left(\hat{x}, x_{0}\right)$ and $\tilde{x}_{\eta}>0$ we immediately find that $x_{\eta}^{*}<\tilde{x}_{\eta}$. The inequality $V_{x}(x, p) \geq$ $J_{x}(x, p)$ for all $(x, p) \in \mathbb{R}_{+}^{2}$ now follows directly from the identity $x_{\eta}^{*}=\operatorname{argmin}\left\{\psi_{\eta}^{\prime}(x)\right\}$ and the monotonicity of $\psi_{\eta}^{\prime}(x)$ on $\left(x_{\eta}^{*}, \infty\right)$. Since $\lim _{x \downarrow 0} V(x, p)=\lim _{x \downarrow 0} J(x, p)=0$ we find by integrating the inequality $V_{x}(x, p) \geq J_{x}(x, p)$ that $V(x, p) \geq J(x, p)$ for all $(x, p) \in \mathbb{R}_{+}^{2}$. Finally, the inequalities $V_{p}(x, p) \geq J_{p}(x, p)$ and $V_{p x}(x, p) \geq J_{p x}(x, p)$ follow from the inequalities $V_{x}(x, p) \geq J_{x}(x, p)$ and $V(x, p) \geq J(x, p)$ and the separability of the values. 